\title{
Plasticidad Cerebral y Hábito en William James: un Antecedente para la Neurociencia Social
}

\author{
Carlos María Alcover y Fernando Rodríguez Mazo \\ Universidad Rey Juan Carlos (Spain)
}

Brain Plasticity and Habit in William James: an Antecedent for Social Neuroscience

William James, in the chapter on the habits of "The Principles of Psychology" (1890) introduced as a key concept of plasticity of brain and nervous system. James could not study this phenomenon experimentally, but his proposal was derived from the results of contemporary research in different fields of Biology and Physiology. Plasticity refers to how learning, skill acquisition, interpersonal and social influences and other contextual variables can influence on the physical structure of the brain, modifying and establishing new relationships and neural circuits that in turn can impair their functioning. This concept was studied experimentally in the late Twentieth Century, and it's a key concept in the current Social Neuroscience, a discipline that seeks to combine and integrate different conceptual and methodological elements from Neuroscience and Social Psychology. This analysis has allowed us, first, to emphasize the meaning and value that James gave to the concept of plasticity in its analysis of habit, and second, to review the meaning of this concept in modern Social Neuroscience, stressing background of the James' hypotheses in the current concept of brain plasticity.

Keywords: brain plasticity, habit, William James, social neuroscience.

En el capítulo dedicado al hábito de los Principios de Psicología (1890) William James introducía como concepto clave la plasticidad del sistema nervioso y del cerebro, un fenómeno que experimentalmente él no podía estudiar, pero que era una consecuencia derivada de los resultados de las investigaciones contemporáneas en diversos campos de la Biología y la Fisiología. La plasticidad hace referencia a cómo el aprendizaje, la adquisición de habilidades, las influencias interpersonales y sociales y otras variables del contexto pueden ejercer un efecto en la estructura física del cerebro, modificándolo y estableciendo nuevas relaciones y circuitos neurales que a su vez alteran su funcionamiento. Este concepto, estudiado experimentalmente a finales del siglo XX, es una de las claves en la actual Neurociencia Social, disciplina que trata de combinar e integrar diferentes elementos conceptuales y metodológicos procedentes de las Neurociencias y de la Psicología Social. Este análisis ha permitido, en primer lugar, destacar el significado y el valor que James otorgó al concepto de plasticidad en su análisis del hábito, y en segundo lugar, revisar el significado de este concepto en la Neurociencia Social, subrayando el papel antecedente de las hipótesis de James en la concepción actual de plasticidad cerebral.

Palabras clave: plasticidad cerebral, hábito, William James, neurociencia social.

Esta investigación ha sido financiada en parte por la Dirección General de Universidades e Investigación, Consejería de Educación de la Comunidad de Madrid, Referencia del Proyecto: S2007/HUM-0413.

Correspondence concerning this article should be addressed to Carlos María Alcover, or Fernando Rodríguez Mazo. Unidad de Psicología Social, Departamento de Psicología, Universidad Rey Juan Carlos. Paseo de los Artilleros, s/n. 28032 Madrid (Spain). E-mails: carlosmaria.alcover@urjc.es - fernando.rodriguez@urjc.es 
William James (1842-1910) incluyó como capítulo IV de su obra Principios de Psicología (James, 1890) el artículo publicado previamente en el número de febrero de 1887 de Popular Science Monthly titulado "El hábito". Se trata de un capítulo sumamente interesante por el carácter anticipador de ideas que también S. Freud y S. Ramón y Cajal van a tratar desde sus posiciones, intereses e investigaciones (Ansermet y Magistretti, 2004; Kandel, 2006). La idea fundamental que aquí nos interesa es la de la plasticidad del sistema nervioso, y en particular, del cerebro, un fenómeno que experimentalmente ninguno de ellos podía estudiar ni demostrar, pero que era una consecuencia derivada de los resultados de las investigaciones contemporáneas en diversos campos de la Biología y la Fisiología.

Hubo que esperar más de medio siglo para que Donald O. Hebb, en su trabajo Organización de la conducta (Hebb, 1949), retomara estas ideas y afirmara que la experiencia modifica las conexiones corticales, de manera que incluso el cerebro de una persona adulta está constantemente cambiando en respuesta a la experiencia. Investigaciones recientes han demostrado que Hebb estaba en lo cierto y que los cambios plásticos en el cerebro se encuentran asociados al aprendizaje y a la memoria, a la adquisición de habilidades, e incluso al establecimiento de adicciones (Gottesman y Hanson, 2005). En la actualidad se ha demostrado que una miríada de factores influyen sobre la plasticidad cerebral: la experiencia pre- y post-natal, los genes, el consumo de drogas, las hormonas, la maduración y el envejecimiento, la dieta, el estrés, o las enfermedades y los accidentes (Kolb, Gibb, y Robinson, 2003). En definitiva, toda experiencia deja una huella. Esta afirmación ha sido confirmada de modo experimental por hallazgos recientes en Neurobiología, que muestran cómo la plasticidad de la red neuronal permite la inscripción de la experiencia, la cual modifica permanentemente las conexiones entre las neuronas, provocando cambios tanto de orden estructural como funcional (Ansermet y Magistretti, 2004). Avances recientes señalan un importante factor implicado en los cambios en el funcionamiento cerebral: el mundo social; de los estudios realizados sobre recuperación de funciones cognitivas tras daños cerebrales se deriva que los procesos de aprendizaje para ser conscientes de cosas nuevas (es decir, para adquirir nuevos qualia) dependen críticamente de la interacción social, en particular de la retroalimentación proporcionada por el entrenador (Frith, 2011). Si bien nos encontramos aún en el comienzo de los estudios experimentales que permitan comprobar el alcance de estos planteamientos, más de un siglo antes de estos hallazgos James ya se sentía estimulado por las hipótesis sobre los fenómenos de plasticidad y su relevancia para el estudio del comportamiento humano.

Los objetivos de este trabajo se dirigen, en primer lugar, al estudio del significado y del valor que James otorga al concepto de plasticidad en su análisis del hábito, y en segundo lugar, a revisar el significado de este concepto en las moder- nas neurociencias, en particular en la Neurociencia Social, subrayando el papel antecedente de las hipótesis jamesianas en la concepción actual de la plasticidad cerebral.

\section{William James sobre el hábito}

James comienza los Principios con dos capítulos sobre el cerebro, a los que sigue otro en el que presenta una teoría del hábito fundamentada en la Fisiología. Su análisis se inicia con una constatación: los seres vivos son manojos de hábitos, los cuales pueden dividirse en dos tipos. Los hábitos que obedecen a una tendencia innata se consideran instintos; aquellos debidos a la educación son denominados habitualmente como actos de razón. Ambos dirigen la mayor parte de la vida, y cualquiera que persiga el estudio del comportamiento de la mente deberá establecer cuáles son sus límites. Los hábitos tienen importantes consecuencias en la vida real de los individuos: simplifican los movimientos requeridos para obtener un determinado resultado, los hace más exactos y disminuye la fatiga; y también reducen la atención consciente con que se ejecutan los actos, automatizando la conducta y haciéndola más fluida, aunque la conciencia es capaz de detectar cualquier desviación y rectificar de inmediato. A esto se añade la posibilidad de cambiar o abandonar hábitos establecidos y adquirir otros nuevos. De alguna manera, ambos tipos de hábitos guardan una simetría con los dos tipos de funcionamiento del cerebro, según James: las funciones inferiores se ocupan del comportamiento automático, mientras que las funciones superiores se ocupan de los procesos intelectuales exclusivamente humanos.

Prosigue James afirmando que en el mundo orgánico, en la materia compuesta, los hábitos son muy variables, tanto en los instintos que varían de un individuo a otro de la misma especie, como en los adquiridos por un mismo individuo que cambian según la experiencia o las exigencias del medio. ¿Pero por qué cambian los hábitos?

"Porque en última instancia se deben a la estructura del compuesto, y sea por fuerza externa o por tensiones internas pueden, de una hora a otra, cambiar esa estructura y hacerla algo diferente de lo que fue. Es decir, pueden hacerlo si el cuerpo es lo suficientemente plástico como para mantener su integridad, y no descomponerse cuando su estructura cede" (James, 1890, p. 86).

En consecuencia, la materia orgánica posee en su naturaleza la capacidad de mantenerse en el cambio, de preservar su integridad a través de los cambios experimentados a lo largo de su existencia. Y esta capacidad, que incrementa poderosamente las posibilidades de adaptación de los organismos, descansa en esta propiedad de la plasticidad. La idea tiene un profundo calado, no sólo para la Biología y para la Psicología, sino también para la integración de posturas filosóficas que habían marcado los debates entre escuelas desde la Antigüedad: la unidad y la estabilidad del mundo 
y de lo existente (por ejemplo, Parménides), frente al cambio y la discontinuidad de lo viviente (por ejemplo, Heráclito). Desde esta nueva perspectiva, es posible la permanencia a pesar de los cambios, y es posible cambiar pese a la estabilidad, y ello es factible gracias al mecanismo de la plasticidad.

James adopta un enfoque dinámico -en el que desempeña un papel relevante el tiempo- en la explicación del mecanismo. Los cambios son más o menos lentos, pues la materia opone resistencia a los efectos modificadores, y sólo gradualmente acepta los cambios, lo cual evita que la materia se desintegre en su totalidad. Una vez que la estructura ha cedido a los cambios y adopta una nueva configuración, la propia inercia actúa como condición para la permanencia tanto de la nueva forma como de los hábitos manifestados y adquiridos en el nuevo estado. Y concluye James:

"Así pues, plasticidad, en la acepción amplia de la palabra, significa poseer una estructura lo suficientemente débil para ceder ante una influencia, pero también lo bastante fuerte para no ceder de golpe. En esta estructura, cada fase de equilibrio relativamente estable se caracteriza por lo que podríamos llamar un nuevo conjunto de hábitos" (James, 1890, pp. 86-87).

Es decir, la materia orgánica es maleable, en ella está inscrita una dinámica dialéctica de estabilidad — cambio gradual - nueva estabilidad - nuevo cambio gradual..., y así sucesivamente, que le permite, simultáneamente, mantener su unidad -su identidad- al tiempo que es susceptible de admitir cambios. En estos planteamientos se pueden rastrear las influencias de las ideas darwinianas de adaptación y cambio evolutivo, de tanto impacto en la naciente psicología norteamericana de finales del siglo XIX (Goodwin, 2009). $Y$ añade James una proposición de gran importancia para el análisis que estamos llevando a cabo:

"La materia orgánica, en especial el tejido nervioso, parece estar dotada con un grado de plasticidad extraordinario; de este modo, podemos enunciar como primera proposición la siguiente: los fenómenos de hábito en los seres vivientes se deben a la plasticidad de los materiales orgánicos de que están compuestos sus cuerpos" (James, 1890, p. 87; subrayado en el original).

Y matiza que el sentido de plasticidad se aplica a la estructura interna y a la forma externa.

Los hábitos simples, prosigue en su análisis James, como cualquier otro acontecimiento nervioso, son, mecánicamente, una simple descarga refleja, de manera que su sustrato anatómico debe ser una vía en el sistema. Por su parte, los hábitos complejos son también simples descargas "concatenadas" en los centros nerviosos, debidas a la presencia en ellos de sistemas de vías reflejas organizadas de modo que unas actúan como estímulos de las otras sucesivamente.

"El único problema -reconoce James- mecánico difícil es explicar la formación de novo de un reflejo o vía simple en un sistema nervioso preexistente. (...) Una vez cruzada una vía, es de esperar que la corriente nerviosa se apegue a la ley que siguen la mayoría de las vías que conocemos, que la ahonde más y más para hacerla más permeable que antes, y esto deberá repetirse con cada nuevo paso de la corriente" (James, 1890, p. 89).

En consecuencia, es fácil imaginar cómo a una corriente que ya ha cruzado una vía le será más fácil cruzarla por segunda vez. Pero aquí se presenta otro escollo:

"¿Qué la hizo cruzarla la primera vez? Para contestar a esta pregunta tendremos que apoyarnos en nuestro concepto general del sistema nervioso como una masa de materia cuyas partes se encuentran continuamente en estados de diferente tensión y que constantemente tiende a igualar sus estados" (James, 1890, p. 89).

Y partir de aquí ya sólo se puede especular acerca del modo en que las corrientes nerviosas a veces se disparen siguiendo vías desacostumbradas (debido a cambios accidentales en los procesos de nutrición, por ejemplo). Estas nuevas líneas, abiertas simplemente por un concurso de probabilidades, al ser cruzadas en repetidas ocasiones, pueden llegar a ser el inicio de un nuevo arco reflejo -de un nuevo hábito-, aunque James confiesa que esta explicación es vaga en grado sumo.

En definitiva, para James las influencias externas que penetran en el cerebro ahondan vías (refuerzan hábitos) o crean otras nuevas (establecen nuevos hábitos). Y dichas influencias externas, en consecuencia, modifican tanto la estructura como el funcionamiento cerebral: este es el pleno sentido de la plasticidad cerebral. Como se verá a continuación, en esencia son los mismos planteamientos que defiende la Neurociencia Social actual, y aunque las explicaciones y los mecanismos que ahora se considera que intervienen en estos procesos son muy diferentes, las ideas básicas coinciden de manera significativa.

Pero en este capítulo de James hay más coincidencias sorprendentes entre antiguas y nuevas ideas. Para apoyar sus afirmaciones de manera más consistente, y demostrando una auténtica honestidad intelectual, James cita a continuación en extenso el trabajo del fisiólogo, naturalista y multifacético científico inglés William B. Carpenter (1813-1885), en particular su libro de 1874, Principles of Mental Physiology... Trece años antes de que James publicara el artículo original sobre el hábito, Carpenter escribía:

"En ninguna parte del organismo humano es tan grande la actividad reconstructora, a lo largo de toda la vida, como en la sustancia ganglionar del cerebro (...) Es, además, un hecho de gran significación que la sustancia nerviosa se distingue muy particularmente por sus facultades de reparación, como se puede comprobar cuando las lesiones (...) de la sustancia nerviosa son reparadas por una reproducción completa del tejido normal" (Carpenter, 1874, cit. en James, 1890, pp. 90-91).

Y añadía Carpenter más explícitamente:

"Porque no hay ninguna razón que nos induzca a ver el cerebro como una excepción del principio general de que cada parte del organismo tiende a formarse de acuerdo como 
el modo en que se ejercita habitualmente, y de que esta tendencia debe ser particularmente vigorosa en el aparato nervioso, en virtud de esa regeneración incesante que es la mismísima condición de su actividad funcional" (Carpenter, 1874, cit. en James, 1890, p. 92).

Es de justicia incluir a W. B. Carpenter entre los antecedentes más significativos del concepto de plasticidad cerebral, ya que a pesar de su carácter especulativo o hipotético, es necesario reconocer su presciencia y su pensamiento anticipador a partir de escasos datos empíricos. Si el propio James escribió que "su obra [de W. B. Carpenter] merece ser llamada trabajo de edificación" (James, 1890, p. 99), 120 años después de James podemos decir como mínimo que resulta sorprendentemente moderna. Finalizando con el capítulo de James, es precisamente la frase de Carpenter de que "nuestro sistema nervioso tiende a formarse de acuerdo con el modo en que se ejercita habitualmente" la que llama poderosamente su atención, pues considera que "expresa en pocas palabras la filosofía del hábito" (James, 1890, p. 92), y le permite concluir el artículo con una serie de aplicaciones prácticas de este principio a la vida humana.

\section{El concepto de plasticidad en la Neurociencia actual}

La idea de un cerebro dinámico, herramienta básica para producir un único individuo (o fenotipo) influenciado por las demandas del entorno, a partir de una combinación genética (o genotipo) determinada, ha interesado a los neurocientíficos desde hace décadas. A pesar de lo evidente que de manera intuitiva podía resultar esta característica de adaptabilidad cerebral, como se manifestó en las ideas de Carpenter, James, Freud, Cajal, Kornorski y Hebb ya mencionadas, sólo los avances técnicos y metodológicos introducidos en los últimos veinticinco años, que permiten observar cambios estructurales y bioquímicos en los componentes pre y postsinápticos, han permitido descubrir hasta qué punto la plasticidad se halla imbricada en un importante número de niveles de la función cerebral. Así, la investigación sobre plasticidad se ha multiplicado de manera exponencial, tratándose en la actualidad de uno de los temas de referencia en las neurociencias.

La plasticidad cerebral (también denominada plasticidad neuronal, cortical, sináptica o, simplemente, neuroplasticidad) se refiere a la formación de aprendizajes y adaptaciones al entorno a través de modificaciones en las estructuras de las redes neuronales. La experiencia pude alterar tanto la función del cerebro (su fisiología) como la organización (su anatomía), y esta experiencia incluye no sólo las influencias externas sino también algunas internas. Así, se observan ejemplos de plasticidad en las remodelaciones del cerebro tras un accidente, durante un periodo de estrés o ansiedad agudo (Viveros, Marco, Llorente, y López-Gallardo, 2007), simultánea y posteriormente a un episodio de adicción, en los procesos de formación de nuevas neuronas (neu- rogénesis) y, fundamentalmente, durante la exposición del individuo a todo tipo de experiencias y aprendizajes, incluyendo los derivados de situaciones sociales.

Los efectos beneficiosos de la expresión de la plasticidad pueden dividirse en tres grandes grupos: necesidad de desarrollo normal del desarrollo postnatal, facilidad para que el sistema nervioso se adapte a las demandas del ambiente y compensación de pérdidas de funcionalidad y reorganización del sistema nervioso para reemplazar dichas funciones perdidas (Møller, 2006). Algunos autores señalan incluso que la profundización de la investigación en plasticidad podrá llevar a las neurociencias a construir mejores cerebros y a reparar los dañados (Kolb, 1995).

De manera genérica puede afirmarse que la potencialidad de cambio del cerebro se basa en las propiedades fisiológicas y anatómicas de la modulación neuronal y alcanza su máximo desarrollo durante la niñez, manteniéndose durante toda la vida como una herramienta eficaz ante los cambios inducidos o el simple deterioro neuronal debido al proceso de envejecimiento del individuo (Baundry, Thompson, y Davis, 1993). Sus bases neuronales se centran, básicamente, en añadir o restar neuronas de una red, sumar o eliminar conexiones entre ellas, cambiar las eficacias sinápticas y/o reducir o modificar la síntesis de proteínas y la actividad de las encimas peptidasas en las neuronas.

Para comprender este proceso de plasticidad es necesario asumir en primer lugar que, a nivel celular, se trata de una propiedad más característica de las sinapsis que de las neuronas. En los primeros estudios documentados, Ramón y Cajal afirmó que los procesos de adaptación y aprendizaje provocaban cambios morfológicos en la eficacia de las conexiones neuronales (Ramón y Cajal, 1913). Más de treinta años después, Kornorski sugirió dos tipos de cambios en las neuronas de un cerebro estimulado: una modificación en la excitabilidad de las neuronas invariante pero transitoria, y un cambio plástico duradero (Kornorski, 1948). Es decir, se hipotetizaba que la condición necesaria para que las neuronas cambiasen era que se activaran, es decir, que se utilizaran. Por último, Hebb sugirió que el lugar más adecuado para encontrar dichos cambios era la conexión entre las neuronas, es decir, las sinapsis (Hebb, 1949). Cuando las sinapsis están activas, estas cambian si las condiciones son adecuadas. De esta forma, para que se produzca plasticidad dos neuronas deben estar casualmente activas, lo que provocaría un fortalecimiento de su sinapsis. Por tanto los cambios que permiten al cerebro funcionar de manera plástica durante el aprendizaje, la memorización, la recuperación de una lesión o la edad se producirían a nivel sináptico. Bien es cierto que desde un punto de vista global, la plasticidad comportamental será el resultado de la suma e interacción de las plasticidades de las sinapsis individuales (Kolb, 1995).

En la actualidad, los principales núcleos de actividad investigadora en plasticidad se diversifican en distintos niveles de análisis: genético-molecular, estructural y funcional. 
A nivel genético-molecular destaca el interés que despiertan en los investigadores los genes dependientes de actividad (activity-dependent), como instrumentos de control de la plasticidad sináptica (Malenka, 2003). Dichos genes se emplean básicamente para la regulación de la estructura interna de las dendritas (Kuhl, 2000). No menos relevante, y aún con mayor protagonismo en la investigación actual es el estudio del papel de la potenciación a largo plazo (LTP) en la plasticidad (Carlson, 2010). La LTP es un proceso molecular y bioquímico consistente en una intensificación duradera en la transmisión de señales entre dos neuronas que resulta de la estimulación sincrónica de ambas. Dicho incremento de la actividad sólo es posible cuando la membrana postsináptica está despolarizada al mismo tiempo que las sinapsis se encuentran activas (Baudry y Lynch, 1993). Un fenómeno similar, denominado LTP asociativa, se produce cuando la estimulación concurrente en el tiempo de una sinapsis débil y otra fuerte ocasiona el fortalecimiento de la primera. Estos dos procesos, junto con sus contrarios de depresión a largo plazo (LTD) asociativa o no (Ito, 1993), se han convertido en una de las bases sobre las que descansan las explicaciones moleculares de la plasticidad al descifrar, al menos en parte, la remodelación de los contactos sinápticos y la formación de nuevas sinapsis. Pero la verdadera importancia de las investigaciones sobre LTP reside en la posibilidad de dar el salto a los niveles de análisis estructural y funcional, al formar parte de un mecanismo más global de formación y estabilización de sinapsis apropiadas, responsable de al menos algunos de los cambios sinápticos propios de la flexibilidad cerebral del aprendizaje y el recuerdo (Baudry y Thompson, 2000).

El siguiente nivel de análisis es la plasticidad estructural. Se ha comprobado que los cambios bioquímicos mencionados suponen modificaciones respecto al número de sinapsis o su morfología (Rose, 1993) o incluso en un aumento de la neurogénesis (Lehmann, Butz, y TeuchertNoodt, 2005). Existen poderosos indicadores que señalan que la neurogénesis está relacionada con la remodelación sináptica, también en cerebros adultos. Esto se debe a que las nuevas neuronas demandan de integración sináptica, y la supervivencia neuronal depende de la integración funcional dentro de las redes neurales existentes (Schaefers, Grafen, Teuchert-Noodt, y Winter, 2010).

Por último, el interés actual se centra en el estudio de las relaciones entre la plasticidad estructural y la funcional. La investigación es muy prolífica en este punto, con resultados relativos a la memoria y al aprendizaje (Rose, 1993), el desarrollo en entornos de privación o enriquecimiento ambiental (Keller, Bagorda, Hildebrandt, y Teuchert-Noodt, 2000), o, por citar tan solo algunos ejemplos, en el rendimiento somatosensorial (Kalisch, Tegenthoff, y Dinse, 2010)

Desde el punto de vista anatómico se han encontrado mecanismos de plasticidad en distintas formaciones y estructuras cerebrales. Son abundantes las investigaciones sobre plasticidad cortical donde se demuestra que importantes sec- ciones de la corteza cerebral de individuos humanos adultos pueden ser remapeadas como resultado de la experiencia. La revisión de la investigación cortical realizada por Steven y Blakemore (2004) aporta datos que prueban la existencia de plasticidad en el córtex motor, el somatosensorial, el visual, el auditivo y el olfatorio. Sin embargo, y por razones aún no clarificadas, existen importantes diferencias individuales respecto a la capacidad de plasticidad, $\mathrm{y}$ es preciso identificar las causas de ciertas disfunciones de la reorganización plástica como el autismo o los diferentes tipos de sinestesia.

Una de las estructuras cerebrales más estudiadas es la formación hipocámpica, la región especializada del córtex límbico localizada en el lóbulo temporal medial. Aquí se descubrieron las LTP y hoy en día se sigue utilizando con asiduidad para investigar este fenómeno debido a sus densas capas de neuronas (Carlson, 2010). Una parte concreta de la formación hipocámpica, el hipocampo propiamente dicho, encargado de la organización de la memoria de los objetos en el espacio, y según algunas teorías, también de la consolidación de recuerdos, es otro de los lugares preferentes del estudio sobre la plasticidad cerebral. Diversas investigaciones (por ejemplo, McEwen, 2004) se han centrado en el análisis de los efectos de distintos tipos de hormonas en su concreta plasticidad funcional, destacando las relacionadas con el estrés psicosocial, que provocan supresión de neurogénesis y una drástica remodelación de las dendritas, tanto a nivel del hipocampo como de la amígdala, otra de las regiones cerebrales próximas, directamente relacionada con las emociones y el control del estrés. El tálamo somato-sensorial, el cuerpo estriado o ya fuera del cerebro, el cerebelo, son tan sólo algunos ejemplos de la diversificación de estructuras de estudio de la plasticidad cerebral.

Una de las principales aportaciones de las investigaciones aplicadas respecto a plasticidad cerebral y comportamental reside en las estrategias de enriquecimiento ambiental, revisadas extensamente por Nithianantharajah y Hannan (2006). El enriquecimiento ambiental se realiza mediante la aplicación de estrategias de estimulación sensorial y/o intelectual tanto en niños como en adultos y provoca cambios a dos niveles. En primer lugar, modificaciones comportamentales: en adultos, mejoras en aprendizaje y memoria; en niños, aumento de las conductas de exploración, disminución del temor asociado a la novedad y reducción de respuestas de estrés exageradas ante estímulos neutros. En segundo lugar, cambios fisiológicos y anatómicos: aumento del peso y grosor del córtex, cambios en la densidad y afinidad de los receptores sinápticos, mayor número de células de glía, aumento del número de neuronas y sinapsis, LTP de sinapsis, e incluso, aumento del tamaño del cerebro, concretamente del hipocampo (para una extensa revisión, Dawson, Sterling y Faja, 2009).

En definitiva, las neurociencias se enfrentan a numerosos retos respecto a la comprensión y a la utilización de la 
plasticidad cerebral. Por una parte, deberán identificarse los mecanismos exactos del cambio plástico y cómo son utilizados por las funciones cerebrales para ajustar el individuo, tanto adulto como en desarrollo, a las demandas de su ambiente. Por otra parte, desde un punto de vista aplicado, será fundamental analizar los mecanismos implicados en las recuperaciones de daño cerebral; recientemente, Mogensen (2011) ha demostrado que la actividad cerebral encargada de la recuperación no necesariamente se encuentra en la misma localización que originalmente era responsable de la experiencia. Esta observación permite plantear dos importantes conclusiones: la experiencia consciente (qualia) puede ser readquirida a través de algún tipo de proceso de aprendizaje, y la naturaleza de la experiencia (qualia) no se encuentra determinada solo por la naturaleza y/o localización de la actividad cerebral que la sustenta (Frith, 2011). Asimismo, será necesario analizar las causas de que el cerebro en desarrollo sea más flexible que el adulto, algo evidente al observador pero de difícil compresión causal. Aunque en las dos últimas décadas se han producido avances significativos, las intuiciones e hipótesis de James formuladas a finales del siglo XIX mantienen su valor heurístico, y el estudio de los mecanismos y de las consecuencias de la plasticidad cerebral será de una gran relevancia para la comprensión del comportamiento humano complejo.

\section{Conclusiones}

Eric R. Kandel, premio Nobel en 2000 por sus investigaciones sobre los mecanismos neurobiológicos de la memoria, afirmaba en sus memorias (Kandel, 2006): "reflexionar con rigor es mucho más valioso que acumular experimentos, especialmente cuando la reflexión hace brotar al menos alguna idea útil" (p. 192). Las reflexiones de William James, de Ramón y Cajal y de Freud, a las que se sumaron las de Kornosky y Hebb, acerca de la plasticidad neuronal y cerebral, han servido para que Kandel y otros muchos investigadores actuales formulen nuevas hipótesis que guíen sus experimentos. Nuevos enfoques, nuevos datos y nuevas teorías que permiten el avance del conocimiento acerca de la fisiología y la anatomía del cerebro, y con él, el de múltiples mecanismos y procesos del comportamiento humano complejo mediante la integración de los diferentes niveles de análisis, desde el neural hasta el social, incluyendo el cultural y el evolutivo (Semin y Echterhoff, 2010)

Entre estos nuevos avances, durante la última década se ha consolidado una nueva disciplina denominada Neurociencia Social (NS) que trata de combinar e integrar diferentes elementos conceptuales y metodológicos procedentes de las neurociencias y de la Psicología Social, con el objetivo de comprender -evitando tanto el dualismo como cualquier tipo de reduccionismo- cómo los procesos neurales, hormonales e inmunológicos influyen en, y son influidos por, los comportamientos y los procesos psicosociales
(Cacioppo y Berntson, 2002, 2006; Decety y Cacioppo, 2010). En otras palabras, la NS pone el énfasis en el modo en que el funcionamiento cerebral influye sobre los procesos sociales y estos, a su vez, influyen sobre el cerebro, utilizando para el estudio de estas relaciones métodos psicosociales y biológicos (Cacioppo, Berntson, y Decety, 2010; Decety y Keenan, 2006; Harmon-Jones y Winkielman, 2007). La NS se ha convertido en pocos años en un activo y excitante campo de investigación, y aunque aún hay muchas cuestiones por resolver y es necesario el desarrollo de una gran cantidad de investigación para responder a aspectos muy complejos de los fenómenos biopsicosociales estudiados, constituye uno de los ámbitos más prometedores de investigación en la actualidad (Grande-García, 2009).

En esta nueva orientación del estudio del comportamiento humano tiene una importancia central el concepto de plasticidad, referida tanto al cerebro como a la conducta social (Alcover, 2008). Como se ha analizado en este trabajo, las consecuencias de la plasticidad implican que el aprendizaje, la adquisición de habilidades, las influencias interpersonales y sociales y otras variables del contexto, pueden ejercer un efecto en la estructura física del cerebro, modificándolo y estableciendo nuevas relaciones y circuitos neurales que a su vez alteran su funcionamiento (Rosenzweig, Breedlove, y Watson, 2005). Quizá en un ejercicio involuntario de "justicia científica", demostrando más de un siglo después las hipótesis de James, los estudios realizados en los últimos quince años sobre la plasticidad sináptica han permitido comprobar la continua modificación de las sinapsis a lo largo de toda la vida, lo que significa que la conducta de un individuo se produce gracias a mecanismos genéticos y del desarrollo que actúan sobre el cerebro; así, los factores ambientales y el aprendizaje hacen aflorar capacidades específicas alterando la eficacia o las conexiones anatómicas de las vías existentes (Kandel, 2000, 2006).

Por ejemplo, las investigaciones de Michael M. Merzenich y su equipo han permitido argumentar que practicar una nueva destreza en las condiciones adecuadas, es decir, de manera consistente con las leyes que gobiernan la plasticidad cerebral, puede cambiar cientos de millones e incluso miles de millones de las conexiones entre las células nerviosas del cerebro humano (Merzenich, Tallal, Peterson, Millar, y Jenkins, 1999). Sus estudios han mostrado que para mantener un cerebro activo no basta con practicar lo que ya se domina, sino que es necesario aprender destrezas nuevas de manera que el cerebro continúe aprendiendo a aprender; de este modo es posible mantener o reabrir el periodo crítico de aprendizaje en adultos (Kilgard y Merzenich, 1998).

Las aplicaciones de sus estudios se han traducido en el diseño de implantes cocleares en niños congénitamente sordos, en técnicas aplicadas a niños con trastornos de aprendizaje para mejorar sus procesos cognitivos y perceptivos, y en niños autistas, así como en la recuperación a través de la reestructuración de los mapas cerebrales de adultos 
con dificultades cognitivas (Merzenich, 2001). Sus experimentos con primates y con humanos han permitido comprobar que el cerebro se estructura mediante una interacción continua con el mundo exterior, y esta influencia no se limita a los órganos y las funciones más expuestas a los estímulos externos, como los sensoriales, sino que también afecta a estructuras y funciones cerebrales más internas. Estos resultados de investigación acerca de la plasticidad del cerebro pueden tener una importancia significativa para la NS: se trata de comprender cómo los estímulos, las experiencias y las relaciones sociales pueden influir en los procesos y en las estructuras cerebrales, (re)configurando su funcionamiento y permitiendo una mejor adaptación a las condiciones del entorno. Esta posible integración de niveles de análisis -desde los factores sociales y ambientales hasta los psicológicos y cognitivos, pudiendo alcanzar incluso al nivel genético- supondría avances relevantes en el conocimiento de los mecanismos que regulan el comportamiento humano, y una posibilidad de potenciar tanto el funcionamiento normal del cerebro y del organismo como de restaurar y recuperar funciones dañadas a lo largo del ciclo vital de las personas, puesto que la plasticidad, al menos en ciertos niveles, se podría mantener durante toda la vida (Alcover, 2008).

En este trabajo hemos analizado los múltiples paralelismos entre las proposiciones de James sobre la plasticidad en su análisis del hábito y los resultados de las investigaciones más recientes realizadas desde las neurociencias, los cuales confirman en gran medida las hipótesis jamesianas. Además de analizar su valor como antecedente directo de la investigación actual en NS, también se ha puesto de manifiesto el papel orientador y el valor heurístico de las formulaciones teóricas -e incluso intuitivas- en la investigación. En los próximos años la NS se centrará en la investigación sobre los procesos de plasticidad en el cerebro y en el comportamiento humano en sus diferentes niveles de análisis. Es muy probable que sus resultados modifiquen buena parte de nuestros conocimientos actuales, además de contar con significativas aplicaciones prácticas destinadas tanto a la mejora de procesos básicos como el aprendizaje y la adquisición de habilidades (Green y Bavelier, 2008), la memoria, la percepción social o las relaciones interpersonales, como al tratamiento y recuperación de diferentes funciones motoras y cognitivas en cerebros dañados (Raskin, 2011), lo que constituye un desafío y un estímulo para la investigación.

\section{Referencias}

Alcover, C. M. (2008). Neurociencia Social: Hacia la integración de las explicaciones sociales y biológicas de la conducta social [Social Neuroscience: Towards the integration of social and biological explanations of social behavior]. En J. F. Morales, C. Huici, A. Gómez, \& E. Gaviria (Coords.), Método, teoría e investigación en psicología social (pp. 187-213). Madrid, España: Pearson Prentice Hall.

Ansermet, F., \& Magistretti, P. (2004). A cada cual su cerebro. Plasticidad neuronal e inconsciente [To each his own brain. Biology of the unconscious]. Buenos Aires, Argentina: Katz Editores, 2006.

Baudry, M., \& Lynch, G. (1993). Long-term potentiation: Biochemical mechanisms. En M. Baudry, R. F. Thompson, \& J. L. Davis (Eds.), Synaptic plasticity. Molecular, cellular, and functional aspects (pp. 87-115). Cambridge, MA: MIT Press.

Baudry, M., \& Thompson, R. F. (2000). Synaptic plasticity: From molecules to behavior. En M. Baudry, J. L. Davis, \& R. F. Thompson (Eds.), Advances in synaptic plasticity (pp. 299319). Cambridge, MA: MIT Press.

Baudry, M., Thompson, R. F., \& Davis, J. L. (Eds.) (1993). Synaptic plasticity. Molecular, cellular, and functional aspects. Cambridge, MA: MIT Press.

Cacioppo, J. T., \& Berntson, G. G. (2002). Social neuroscience. En J. T. Cacioppo, G. G. Berntson, R. Adolphs, C. S. Carter, R. J. Davidson, M. K. McClintock, ... S. E. Taylor (Eds.), Foundations in social neuroscience (pp. 1-9), Cambridge, MA: MIT Press.

Cacioppo, J. T., \& Berntson, G. G. (2006). A bridge linking Social Psychology and the Neurosciences. En P. A. M. van Lange (Ed.), Bridging social psychology: The benefits of transdisciplinary approaches (pp. 91-96). Hillsdale, NJ: Erlbaum.

Cacioppo, J. T., Berntson, G. G., \& Decety, J. (2010). Social neuroscience and its relationship to social psychology. Social Cognition, 28, 675-685. http://dx.doi.org/10.1521/soco.2010.28. 6.675

Carlson, N. R. (2010). Physiology of Behavior (10 ${ }^{\mathrm{a}}$ Ed.). Boston, MA: Pearson Education.

Carpenter, W. B. (1874). Principles of mental physiology, with their applications to the training and discipline of the mind and the study of its morbid conditions. Nueva York, NY: Appleton.

Dawson, G., Sterling, L., \& Faja, S. (2009). Autism. Risk factors, risk processes, and outcome. En M. de Haan \& M. R. Gunnar (Eds.), Handbook of developmental social neuroscience (pp. 435-458). Nueva York, NY: The Guildford Press.

Decety, J., \& Cacioppo, J. T. (2010). Frontiers in human neuroscience: The golden triangle and beyond. Perspectives on Psychological Science, 5, 767-771. http://dx.doi.org/10. $1177 / 1745691610388780$

Decety, J., \& Keenan, J. P. (2006). Social neuroscience: A new journal. Social Neuroscience, 1, 1-4. http://dx.doi.org/10.1080/17 470910600683549

Frith, C. D. (2011). What brain plasticity reveals about the nature of consciousness: Commentary. Frontiers in Psychology, 2(87), 1-3. http://dx.doi.org/10.3389/fpsyg.2011.00087

Goodwin, C. J. (2009). Historia de la psicología moderna [A history of modern psychology]. México: Limusa Wiley.

Gottesman, I. I., \& Hanson, D. R. (2005). Human development: Biological and genetic processes. Annual Review of Psychology, 56, 263-286. http://dx.doi.org/10.1146/annurev.psych.56.0911 03.070208 
Grande-García, I. (2009). Neurociencia social: El maridaje entre la psicología social y las neurociencias cognitivas. Revisión e introducción a una nueva disciplina [Social neuroscience: The marriage between social psychology and cognitive neurosciences. A review and an introduction to a new discipline]. Anales de Psicología, 25, 1-20.

Green, C. S., \& Bavelier, D. (2008). Exercising your brain: A review of human brain plasticity and training-induced learning. Psychology and Aging, 23, 692-701. http://dx.doi.org/10.1037/a 0014345

Hebb, D. O. (1949). Organización de la conducta [The organization of behavior]. Madrid, España: Debate, 1985.

Harmon-Jones, E., \& Winkielman, P. (2007). A brief overview of Social Neuroscience. En E. Harmon-Jones \& P. Winkielman (Eds.), Social meuroscience. Integrating biological and psychological explanations of social behavior (pp. 3-11). Nueva York, NY: Guilford Press.

Ito, M. (1993). Cerebellar mechanism of long-term depression. En M. Baudry, R. F. Thompson, \& J. L. Davis (Eds.), Synaptic plasticity. Molecular, cellular, and functional aspects (pp. 117128). Cambridge, MA: MIT Press.

James, W. (1890). Principios de psicología [Principles of psychology]. México: Fondo de Cultura Económica, 1989.

Kalisch, T., Tegenthoff, M., \& Dinse, H. R. (2010). Repetitive electric etimulation elicits enduring improvement of sensorimotor performance in seniors. Neural Plasticity, 2010, 1-11, http://dx.doi.org/10.1155\%2F2010\%2F690531

Kandel, E. R. (2000). Mecanismos celulares del aprendizaje y sustrato biológico de la individualidad [Cellular mechanisms of learning and biological substrate of individuality]. En E. R. Kandel, J. H. Schwartz, \& T. M. Jessel (Eds.), Principios de neurociencia [Principles of neuroscience] (pp. 1247-1279). Madrid, España: McGraw-Hill, 2001.

Kandel, E. R. (2006). En busca de la memoria. El nacimiento de una nueva ciencia de la mente [In search of memory. The emergence of new science of mind]. Buenos Aires, Argentina: Katz Editores, 2007.

Keller, A., Bagorda, F. Hildebrandt, K., \& Teuchert-Noodt, G. (2000). Effects of enriched and of restricted rearing on both neurogenesis and synaptogenesis in the hippocampal dentate gyrus of adult gerbils (Meriones unguiculatus). Neurology Psychiatry and Brain Research, 8, 101-108.

Kilgard, M. P., \& Merzenich, M. M. (1998). Cortical map reorganization enabled by nucleous basalis activiy. Science, 279, 1714-1718. http://dx.doi.org/10.1126/science.279.5357.1714

Kolb, B. (1995). Brain plasticity and behavior. Mahwah, NJ: Lawrence Erlbaum.

Kolb, B., Gibb, R., \& Robinson, T. E. (2003). Brain plasticity and behavior. Current Directions in Psychological Science, 12, 15. http://dx.doi.org/10.1111/1467-8721.01210

Kornorski, J. (1948). Conditioned reflexes and neuron organization. Cambridge, MA: Cambridge University Press.

Kuhl, D. (2000). Learning about activity-dependent genes. En M. Baudry, J. L. Davis, \& R. F. Thompson (Eds.), Advances in synaptic plasticity (pp.1-31). Cambridge, MA: MIT Press.
Lehmann, K., Butz, M., \& Teuchert-Noodt, G. (2005). Offer and demand: Proliferation and survival of neurons in the dentate gyrus. European Journal of Neuroscience, 21, 3205-3216. http://dx.doi.org/10.1111/j.1460-9568.2005.04156.x

Malenka, R. C. (2003). Synaptic plasticity. En K. L. Davis, D. Charney, J. T. Coyle, \& C. Nemeroff (Eds.), Neuropsychopharmacology: The fifth generation of progress (pp. 147-157). Nashville, TN: American College of Neuropsychopharmacology.

McEwen, B. S. (2004). How sex and stress hormones regulate the structural and functional plasticity of the hippocampus. En M. S. Gazzaniga (Ed.), The cognitive neurosciences (Vol.3, pp. 171-181). Cambridge, MA: MIT Press.

Merzenich, M. M. (2001). Cortical plasticity contributing to childhood development. En J. L. McClelland, \& R. S. Siegles (Eds.), Mechanisms of cognitive development: Behavioral and neural perspectives (pp. 61-83). Mahwah, NJ: Lawrence Erlbaum.

Merzenich, M. M., Tallal, P., Peterson, B., Miller, S., \& Jenkins, W. M. (1999). Some neurological principles relevant to the origins of — land the cortical plasticity-based remediation of developmental language impairments. En J. Grafman \& Y. Christen (Eds.), Neuronal plasticity: Building a bridge form the laboratory to the clinic (pp. 169-187). Berlín, Alemania: Springer-Verlag.

Møller, A. R. (2006). Neural plasticity and disorders of the nervous system. Cambridge, MA: Cambridge University Press.

Mogensen, J. (2011). Reorganization of the injured brain: Implications for studies of the neural sub-strate of cognition. Frontiers in Psychology, 2, 1-7. http://dx.doi.org/10.3389/fpsyg. 2011.00007

Nithianantharajah, J., \& Hannan, A. J. (2006). Enriched enviroments, experience-dependent plasticity, and disorders of the nervous system. Nature Reviews Neuroscience, 7, 697-709. http://dx.doi.org/10.1038/nrn1970

Ramón y Cajal, S. (1913). Estudios sobre la degeneración y regeneración del sistema nervioso. Tomo 1: Degeneración y regeneración de los nervios [Studies on the degeneration and regeneration of the nervous system. Vol. 1: Degeneration and regeneration of nerves]. Madrid, España: Imprenta de hijos de Nicolás Moyá.

Raskin, S. A. (Ed.) (2011). Neuroplasticity and rehabilitation. Nueva York, NY: Guilford Press.

Rose, S. P. R. (1993). Synaptic plasticity, learning, and memory. En M. Baudry, R. F. Thompson, \& J. L. Davis (Eds.), Synaptic plasticity. Molecular, cellular, and functional aspects (pp. 209229). Cambridge, MA: MIT Press.

Rosenzweig, M. R., Breedlove, S. M., \& Watson, N. V. (2004). Biological psychology: An introduction to behavioral, cognitive, and clinical neuroscience. Sunderland, England: Sinauer Associates.

Schaefers, A. T. U., Grafen, K., Teuchert-Noodt, G., \& Winter, Y. (2010). Synaptic remodeling in the dentate gyrus, CA3, CA1, subiculum, and entorhinal cortex of mice: Effects of deprived rearing and voluntary running. Neural Plasticity, 2010, 1-11, http://dx.doi.org/10.1155/2010/870573 
Semin, G. R., \& Echterhoff, G. (eds.) (2010). Grounding sociality. Neurons, mind, and culture. London, England: Psychology Press.

Steven, M. S., \& Blakemore, C. (2004). Cortical plasticity in the adult human brain. En M. S. Gazzaniga (Ed.), The cognitive neurosciences (Vol.3, pp. 1243-1254). Cambridge, MA: MIT Press.
Viveros, M. P., Marco, E. M., Llorente, R., \& López-Gallardo, M. (2007). Endocannabinoid system and synaptic plasticity: Implications for emotional responses. Neural Plasticity, 2007, 1-12. http://dx.doi.org/10.1155/2007/52908

Received September 30, 2011 Revision received March 16, 2012 Accepted March 20, 2012 